\title{
PEDRO LASTRA, UN POETA POST-GUTENBERG TAMBIÉN Y A SU MANERA ${ }^{1}$
}

\author{
Luis Correa-Díaz \\ University of Georgia \\ Athens, Georgia, Estados Unidos \\ correadiaz@hotmail.com
}

With each change, I change too

"Elegia" - New Order

https://www.youtube.com/watch?v=omzJvwYO440

Como Borges, lo mejor de Lastra está en su poesía. Este es un juicio subjetivo, claro, como todos los literarios al final del día, pero sé que hay otras voces que me acompañan en el sentimiento. Por supuesto que el poeta es y ha sido, como un artista renacentista, proteico en la escritura y en la palabra. Allí están sus ensayos y su magisterio, ambos notables, guías de perplejos y ávidos lectores, sin olvidar su arte de la amistad -algo esto último que quedó dicho en el prólogo a una colección de estudios dedicados a su obra poética: Arte de vivir (2006), editado junto a Silvia Nagy-Zekmi de Villanova University.

Pero volviendo a su poesía, que se ha estudiado y celebrado por partes iguales ${ }^{2}$, lo que aquí quisiera dejar anotado -en brevísimas palabras para este

\footnotetext{
Esta nota fue leída (a nombre mío por Marcelo Rioseco de la University of Oklahoma) en las Jornadas en homenaje que la Facultad de Letras y el CELICH de la Pontificia Universidad Católica de Chile organizó en el mes de junio del 2019 para reconocer la larga trayectoria literaria, magisterial y académica de Pedro Lastra.

2 Y de la que se ha dicho, por ejemplo y citando un texto reciente, "La hora de todos", de Marcelo Pellegrini, su prólogo a Cuaderno de la doble vida (2019), que "es una sosegada
} 
congreso que lo honra- es el Lastra post-Gutenberg y ese su reconocimiento de ser partícipe -como el que más de todos nosotros en estos momentos de nuestra historia/cultura como especie- de esta nuestra "doble vida". No aquella que es sinónimo de extranjería, la suya en los Estados Unidos por una larga temporada, mientras ejerciera su docencia universitaria en SUNY-Stony Brook. No esta ni otras 'dobles vidas', desdoblamientos de la experiencia humana y literaria, a las que sus poemas convierten en centro o, a veces, simplemente en alusión en sus meditaciones, puesto que sus versos siempre elaboran su decir, cuasi silencioso, como un acto meditativo.

Entre estas otras 'dobles vidas' -y su lector/a ya las habrá ido enumerando a través de sus lecturas lastrianas-, una que se impone con mayor rigor, en la medida que su obra y su mester meditan cada vez más sobre sí mismos, se puede localizar, como un ejemplo apenas aquí, en un poema "inédito" antes de la última entrega, el titulado "La hora malvenida":

La primavera se despide y muere,
con ella vamos como flores inciertas
llamadas por la tierra.
Y yo sigo pensando
que alguien a quien amo
ha desaparecido otra vez de mi lado.
Y de pronto comprendo
que más bien soy yo mismo el desterrado
desde hace mucho tiempo,
yendo y viniendo entre las hojas secas
sin huellas ni señales $(C D V, 125)$.

Aquí el poeta se desdobla, al reflexionar/experimentar la sombra del otro que desaparece de su radar (= "lado", vista, presencia...), en su propia sombra, en su fantasma... Y si leemos, cuando leemos, "desterrado" y nos sugiere 'enterrado' también, de pronto comprendemos la consonancia de todas esas 'dobles vidas' en la poesía de Lastra. Tal vez se puedan definir como la nopertenencia y la im/permanencia, vocablos y conceptualizaciones filosóficopoéticas que no le son ajenas al poeta.

meditación sobre el tiempo, de lo que se desprende necesariamente una reflexión sobre la muerte", y también "una partitura musical compuesta para que se interprete sin estrépito y con disimulo, 'a la sordina"” (13). 
De alguna u otra manera, estas han sido y seguirán siendo, propia y justamente, preocupaciones del creador y sus críticos. Sin embargo, lo que quiero dejar anotado -y que no deja de tener relación estrecha con lo anteriores la introducción de un factor inesperado en su poética, su reconocimiento de (y por ende, su participación en...) lo que J. Hillis Miller llama "la doble vida" en su "The Poetics of Cyberspace: Two Ways to Get a Life" (2007): el contraste divergente $y / 0$ convergente entre "the poetics of print works and the poetics of works in cyberspace" (277); nuestra vida cultural en dos mundos, el impreso y el digital ${ }^{3}$. Aparición tardía la de esta noción en la poesía lastriana, pero no por eso irrelevante. Por el contrario, su demora (y su por poco no-mención, no-interés...) es del todo justificada en sí misma y la hace más notoria en su carácter único. Lastra se ha hecho (y se ha decidido a ser) testigo de este gran cambio en la cultura y, por ende, en la multidimensional empresa literaria.

El poema en cuestión es el siguiente, que resulta ser uno de los fragmentos textuales de que está compuesto su libro Transparencias (2014):

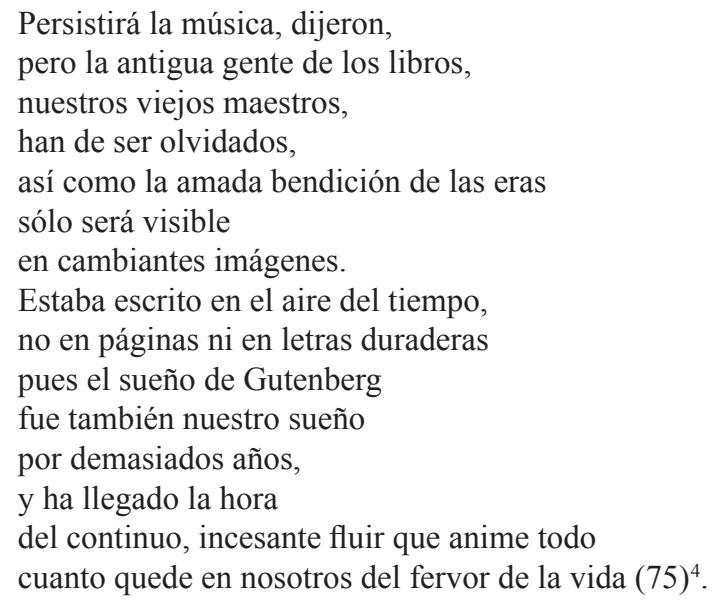

3 No se olvide, eso sí, lo que dice Daniel Morris, citando al poeta estadounidense Kenneth Goldsmith (1961), en su Not Born Digital (2016), que de hecho, y en su mayor parte, nosotros no hacemos realmente "computer" sino que "Internet", y en el ámbito de las letras/ humanidades todavía las excepciones son precisamente eso y pocas.

$4 \quad$ Viene antologado en Cuaderno de la doble vida (2019) en la página 100. 
No voy a interpretar para (/con ni por) ustedes este poema, esta "partitura", como diría Pellegrini; mucho me temo que lo haría con estrepitoso fraseo. Solo me he propuesto dejarlo señalado como documento, mínimo pero necesario y significativo, en el despliegue y repliegue de su poética. No se podría hablar de ésta en su totalidad sin mencionar este aspecto, esta inflexión inesperada, vuelvo a decir. Y no es que tal cosa convierta al poeta en un actor interesado en los nuevos medios, ni en las prácticas digitales actuales de la literatura, ni que la suya esté situada entre la cultura de la información y la literaria, ni tampoco que su poesía se perfile en el ciberespacio -aunque está presente, de todos modos, como quien más en nuestros días... Ni siquiera que se le vea inclinado a elucubrar sobre el asunto de la IA (Inteligencia Artificial, producto de la computación, claro, hasta ahora...), como parece oírse, con cierto dejo tradicionalmente contrariado, en uno de los fragmentos poéticos de Transparencias (y no hay más de estas cosas en Lastra hasta donde llega mi conocimiento):

Con quién hablan las máquinas parlantes?

Hablan consigo mismas, se despeñan

las voces en el río de sus ecos:

rodados de un barranco, las figuras

humanas sepultadas en su seno

son el solo y el mismo mismo eco" (37).

No hay más de ello en su obra, y creo que, a riesgo de equivocarme, no lo habrá.

Lastra insistirá toda su vida en el (formato, la plataforma cultural/ tecnológica) libro. Y eso está muy bien y para él, como para muchos de las generaciones pre-digitales -como igualmente para muchos de las digitales que o no abrazaron este '(inter)media(lity) concern for poetics' o vuelven después de haberlo hecho a la 'print culture'-, no tendría por qué ser distinto. Recuérdese que el libro y su tecnología, pese a lo que se predijo por los que hicieron, y todavía hacen aunque en menor grado, de 'champions' de la "digital media as a newer and better cultural platform", no ha desaparecido ni lo hará hasta donde cabe imaginar sin especulaciones mal razonadas ${ }^{5}$.

Se sabe que el libro, pese a lo anunciado de su muerte/desaparición, sigue siendo la plataforma de validación y, si llega el caso, de consagración de un 
autor. Eso me llevó a citar el poema entero de Lastra en la "Nota preliminar" a Novissima verba... (2019), que versa sobre los 'avatares' (en su doble sentido) de poesía digital/electrónica, cibernética latinoamericana, pues como documento tal tiene un valor literario-arqueológico -e incluso el de un viaje inverso al futuro-, como lo han tenido desde hace tiempo ya, y de manera inversa, las dos "Hada[s] Cibernética[s] de Carlos Germán Belli de los años sesenta -siendo así que "bastan estos dos poemas para situar a Belli en el advenimiento de la poesía electrónica y/o digital, no tanto como género sino que, más bien, en su meta-poética, es decir, en la reflexión sobre el medio" o ciberespacio que ya se veía venir en ese entonces (Correa-Díaz 173). Así, una mirada tal, la del mirar lastriano desde el futuro (hacia) nuestro presente -como el de Belli, el futuro desde un presente que se siente manriqueñamente ido-, era una de las acciones teóricas del libro mío, y por lo mismo no podía no considerar a ambos poetas (maestros) como puntos de referencia en una historia que tal vez se negaría, en sus prisas, a reconocerlos como parte de ella.

El valor de este documento, en su libro que fue Transparencias (2014) y en cualesquiera de los libros antológicos en que aparezca, como ahora lo hizo en Cuadernos de la doble vida (2019), reside en que muestra a Lastra con la dignidad de un maestro de toda la vida, reconociendo lo inevitable: la realidad de la era post-Gutenberg, y sin (de)cantar sus novedades, ni panegírica ni apocalípticamente. Se trata de un documento culturalmente elegíaco y testamental. Pero, sobre todo, de un leve indicio, muy lastriano, de silenciosa participación del poeta, sin hacer de él nada de lo que ya no era, en esta, contradictoria a veces, convergencia de culturas que describiera Henry Jenkins en el 2007.

Si bien es cierto que dije recién que no ofreceré una interpretación del poema per se, el que se explica a sí mismo como documento cultural de una época como la nuestra, quisiera dejar anotada una reflexión final sobre la elegía que le da forma y que, por lo mismo, cumple una función testamentaria dentro de la poética lastriana, por la que se nos transfiere a sus lectores su legado.

Ya en 1994, Sven Birkerts, en su The Gutenberg Elegies, subtitulado The Fate of Reading in an Electronic Age, declaraba con claridad y nostalgia que el "influjo de las comunicaciones electrónicas" y la creciente formatización tecnológica de la información había instalado en la cultura humana un cambio, una "metamorphosis" irreversible. El mundo de nuestras acciones y deseos había acelerado su velocidad a tal punto que no se reconocería más en aquel viejo y lento hacer de antes. Dentro de este panorama, Birkets pronuncia una sentencia que nos importa mucho aquí -y que podría citarse como una 
anticipación del millar de debates al respecto que vendrían al final de aquella década y las siguientes. "The stable hierarchies of the printed page -one of the defining norms of the world- are being superseded by the rush of impulses through freshly minted circuits". Y, en particular, sabiendo que este cambio es solo una de las transformaciones a todo nivel, donde el impulso económico tiene un liderazgo incuestionable, como siempre en nuestra historia, conviene recordar esta observación clínica: "The displacement of the page by the screen is not yet total (as evidenced by the book you are holding) -it may never be total- but the large-scale tendency in that direction has to be obvious to anyone who looks" (3). No ha sido total, ni lo será quizás, como más tarde lo describiera Miller en su "thought experiment" de la "doble vida" que llevamos hoy, mencionado más arriba. Pero ha sido indiscutible y de alcances previsibles e imprevisibles a la vez. Lo mismo puede decirse de la jerarquía (social, estética, espistemológica y axiológica) y la estabilidad que tuvo la literatura (tanto lectura como escritura) en aquel mundo, incluso hasta llegar a postular algo así como "the fading of literature" que, según Miller, puede parecer extremo, pero no menos real como un pronunciamiento sobre el estado de la cuestión ${ }^{6}$. Pues, la verdad sea dicha sin temor ni dolor..., y lo digo con conocimiento (profesional) de causa: ..."the old act of slowly reading a serious book becomes an elegiac exercise" (Birkerts 6, cursivas mías porque quisiera señalar que ambos adjetivos son signos de su tiempo y también diferenciales de la tecno-reconversión de nuestras vidas que siguen siendo lo que eran, pero ya no como eran porque han sido tecnológicamente expandidas). Juanpere en su artículo sobre arte/literatura expandida explica que

la noción de literatura expandida [...] se ha usado repetidamente en los últimos años, especialmente en el campo de las artes visuales, para designar un tipo de práctica artística que se propone investigar nuevas formas de escritura más allá del soporte libro y desvinculada

\footnotetext{
"These teletechnologies have gradually displaced not only magic stage assemblages but also that other fading form of secular magic: literature. Cinema, television, CDs, VCRs, MP3 gadgets, DVD players, computers, and the Internet have become our dominant far-seeing and far-hearing conjurers, sorcerers, prestidigitators, animators of talking heads. [...] Their poetics, especially the poetics of cyberspace, have incalculable power to determine ideological belief, just as books did in the age of the book, the paper period" (Miller 272).
} 
del lenguaje verbal, presentándose como un lugar de expansión entre la visualidad y la textualidad $(103)^{7}$,

aunque, evidentemente y como se ha visto en la medida en que la multimedialidad se hace más compleja y abarcadora, la expansión no solo se consigue a través de la visualidad, igualmente por medio de lo kinético y otras aplicaciones tecnológicas -lo que incluye, como puntualiza Juanpere, "nuevos modos de autoría" y "nuevos lectores" (109-110); pero lo que sí está claro hoy es que expandir en literatura es desafiar esa estabilidad/dominio literario del texto $\mathrm{y}$, en sentido más amplio, la primacía intelecto-cultural del libro, al menos en su versión gutenberguiana ${ }^{8}$.

Entonces, ahí está en el 2014 la elegía de Lastra a "la antigua gente de los libros", a esos "nuestros viejos maestros" que "han de ser olvidados", ese documento que da cuenta de la situación. Y también allí está lo que he llamado -con un afecto renovado por el maestro-, el 'Lastra, poeta postGutenberg': ... "el sueño de Gutenberg / fue también nuestro sueño / por demasiados años". En esta última cláusula adverbial de tiempo aparece el Lastra en pleno uso de sus poderes, en cuanto se detecta aquí su capacidad de historiógrafo cultural de su oficio y (de las edades) del libro: esa pretendida estabilidad y lugar jerárquico de la página impresa era y fue un sueño -aunque haya que tener en cuenta que la pantalla es en mucho otra página, así como ésta era la pantalla de la gente de los libros, como sostengo en un capítulo de Novissima verba...-, y lo hizo tempranamente, para empezar la discusión en torno al tema, Perloff en su citadísimo ensayo "Screening the Page / Paging the Screen" (2006), y hace poco - para mostrar aquí extremos de un espectro- Borsuk en su The Book (2018). Es más, hay una situación paradójica que no terminamos de aceptar o entender, pero

the infinite scrolling text, our digital Reading environments maintain codex-like features, from bookmarking and page turning on-screen

7 Véase el artículo de Kozak también.

Adell señala también hacia ese cuestionamiento de las concepciones ideológicas tradicionales de la literatura -y por extensión, su vehículo libro-, cuando dice: "[n]o sólo porque el recurso a la informática modificaba profundamente los procesos de creación de obras literarias, y porque se producía una mutación radical de los vínculos entre el "autor" inicial, el 'poeta' y su obra, así como el papel que desarrollaba el lector en los procesos de lectura, sino también porque podían poner en cuestión algunas concepciones ideológicas previas sobre la literatura" (139). 
to iPad cases that simulate hardback books. So how do we define the book when the [ancient] tablet and scroll have come back to us in full force? (Borsuk xi).

Entonces, no hay más que reconocer que el libro -y no solo el gutenberguiano, el que nos dio a muchos, por varios siglos, la idea de una permanencia de un artefacto que en sí mismo no ha sido sino un capítulo en una historia más rica que la de un sueño voluntarioso- es una tecnología siempre en transformación y que, por lo mismo, su desaparición carece de verosimilitud. Como insiste Borsuk con exactas palabras: "Humans' interaction with language and literature necessitates certain kinds of portables reading experiences." Por eso, y no por nada esotérico, es que parece "only natural that the book should grow and change with us" (xiv).

De este modo, en este reconocimiento lastriano que se hace notar aquí -tal cual lo hiciera en sus propios términos Birkerts, como se dijera anteriormente- reside no solo un llanto por lo ido, sino que también, y necesariamente, la salida hacia el futuro propia de una elegía completa o, si se quiere, la transformación del lamento en una exaltación de la fuerza vital de lo existente -antropológicamente entendido. Por eso es que el poeta no tiene otra opción, consciente de ello y en aceptación total, que terminar su poema de esta manera: " $y$ ha llegado la hora / del continuo, incesante fluir que anime todo / cuanto quede en nosotros del fervor de la vida" (75). Pero, no solo acaba así, Lastra había empezado en ese tono, indicando la dirección única, a la vez que ofrecía el único consuelo posible (el que todavía a esa voz elegíaca del comienzo del poema no le convencía del todo, presintiendo, sin embargo, su inapelable mandato): "Persistirá la música, dijeron"...-dejo abierta la pregunta sobre quiénes fueron los que eso dijeron, pero el lector sabe que toda la poesía de Lastra es una respuesta a tal interrogante; lo mismo vale para el asunto de que sea "la música" lo (único) que "persistirá", pero ya no hay tiempo para desarrollar esto en estas páginas, aunque, repito, su lector/a se ha encontrado con poemas suyos que se la explican. No obstante, cabe también proponer que la música, sin querer simplificar ni emparentar gratuitamente, es eso mismo que Rubén Darío sonetizó en "Ama tu ritmo" (1901); ese ritmo vital con el que impregnamos los instrumentos, incluido el lenguaje (literario), con los cuales tocamos nuestra música de siempre.

No podía ser de otra manera, la poética lastriana que se encarga de meditar sobre la cuestión (y la percepción, porque es eso y no otra cosa) que nos hace sentirnos divididos entre la permanencia (el sueño de..., el deseo de...) y la 
impermanencia (la aceptación de..., la sabiduría sobre...) -sin querer abrir en este momento el capítulo del gran tema religioso, filosófico, científico de los 'cambios' en su poesía-, tenía que hacerse cargo de esta particular "doble vida" que llevamos hoy ${ }^{9}$. Y mi función aquí no ha sido otra sino la de señalar el lugar de este documento en la obra de Lastra y en el tiempo de nuestra cultura humana en general.

\section{BIBLIOGRAFÍA}

Adell, Joan-Elies. "Las palabras y las máquinas. Una aproximación a la creación poética digital”. Ed. Domingo Sánchez-Mesa. Literatura y cibercultura. Madrid: Arco/Libros, S.L., 2004. 267-296.

Agamben, Giorgio. "Réquiem para los estudiantes". Artillería Inmanente. 23/5/20. Noblogs. https://artilleriainmanente.noblogs.org/?p=1514

Birkerts, Sven. The Gutenberg Elegies: The Fate of Reading in an Electronic Age. 1994. NY: Farrar, Strauss and Giroux, 2006.

Borsuk, Amaranth. The Book. Cambridge, MA: The MIT Press, 2018.

Correa-Díaz, Luis. Novissima verba. Huellas digitales, electrónicas y cibernéticas en la poesía latinoamericana. Santiago: RIL Editores/Academia Chilena de la Lengua, 2019.

Correa-Díaz, Luis y Silvia Nagy-Zekmi, eds. Arte de vivir: acercamientos críticos a la poesía de Pedro Lastra. Santiago de Chile: RIL Editores, 2006.

Jenkins, Henry. Convergence Culture. Where the Old and the New Collide. NY: University of New York Press, 2007.

Juanpere, Paula. "Del arte expandido a la literatura expandida. Una aproximación a la posibilidad de la expansión de lo literario en las artes visuales contemporáneas". $452 * F$. Revista de Teoría de la Literatura y Literatura Comparada 119 (2018): 102-113. https:// www.452f.com/index.php/juanpere

$9 \quad \mathrm{Y}$ en este sentido habría que decir que el poema de Lastra no es un réquiem (entendido como himno funerario), ni él se asemeja en esto a la postura que tiene Giorgio Agamben en un texto ("Réquiem para los estudiantes", 23 de mayo del 2020) reciente sobre las transformaciones a que la educación [otra de nuestras formas musicales, digo yo] parece verse sometida post-COVID-19, lo que podría llamarse su virtualización obligada y que podría convertirse en su futuro hasta donde podemos ver, cosa que el filósofo lamenta, en síntesis, debido a la pérdida del valor, no solo pedagógico, de la proximidad física. Razón hay en este lamento, pero también un no aceptar los cambios. Agrego esta nota al revisar este artículo sobre Lastra en plena pandemia y para mostrar cómo se toman posiciones, en particular por generaciones de la print culture, frente a una percibida hegemonía contemporánea de las tecnologías digitales y sus supuestos efectos negativos o no. 
Kozak, Claudia. "Literatura expandida en el dominio digital". El Taco en la Brea 4/6 (2017): 220-245.

Lastra, Pedro. Cuaderno de la doble vida. Prólogo de Marcelo Pellegrini. Valparaíso, Chile: Editorial Pfeifter, 2019.

Transparencias. Ilustraciones de Mario Toral. Santiago, Chile: Editorial Pfeiffer, 2014.

Miller, J. Hillis. "The Poetics of Cyberspace: Two Ways to Get a Life". Ed. Louis Armand, Louis. Contemporary Poetics. Evanston, IL: Northwestern University Press, 2007. 256-278.

Morris, Daniel. Not Born Digital. Poetics, Print Literacy, New Media. NY: Bloomsbury, 2016.

Perloff, Marjorie. "Screening the Page / Paging the Screen. Digital Poetics and the Differential Text". New Media Poetics: Contexts, Technotexts, and Theories. Eds. Adelaide Morris and Thomas Swiss. Cambridge and London: MIT Press, 2006. 143-164. 